\title{
1 Machine Learning in Multi-Omics Data to Assess Longitudinal Predictors of
}

\section{Glycaemic Health}

4 Laurie Prélot ${ }^{1}$, Harmen Draisma ${ }^{1}$, Mila D. Anasanti ${ }^{1}$, Zhanna Balkhiyarova ${ }^{1}$, Matthias Wielscher ${ }^{2}$, Loic

5 Yengo $^{3}$, Beverley Balkau $^{4,5}$, Ronan Roussel ${ }^{6,7}$, Sylvain Sebert ${ }^{2,8,9}$, Mika Ala-Korpela ${ }^{10-15}$, Philippe

6 Froguel ${ }^{1,16}$, Marjo-Riitta Jarvelin $2,8,9,17,18$, Marika Kaakinen ${ }^{1,19,20 \#}$, Inga Prokopenko ${ }^{1,20 \#^{*}}$

${ }^{1}$ Section of Genomics of Common Disease, Department of Medicine, Imperial College London, London

W12 ONN, United Kingdom;

$10{ }^{2}$ Department of Epidemiology and Biostatistics, School of Public Health, Imperial College London,

11 London W2 1NY, United Kingdom;

$12{ }^{3}$ Institute for Molecular Bioscience, The University of Queensland, 306 Carmody Road,

13 St Lucia Qld 4072, Australia;

$14{ }^{4}$ Inserm, CESP Center for Research in Epidemiology and Public Health, U1018, Villejuif, France;

15 5Univ Paris-Saclay, Univ Paris Sud, UVSQ, UMRS 1018, UMRS 1018, Villejuif, France;

$16{ }^{6}$ Inserm U1138, Centre de Recherche des Cordeliers, Paris, France;

17 'University Paris Diderot, Sorbonne Paris Cite, Paris, France; Diabetology, Endocrinology and Nutrition

Department, DHU FIRE, Hopital Bichat, AP-HP, Paris, France;

${ }^{8}$ Center for Life Course Health Research, University of Oulu, Fl-90014 University of Oulu, Finland;

${ }^{9}$ Biocenter Oulu, University of Oulu, Fl-90014 University of Oulu, Finland;

$21{ }^{10}$ Systems Epidemiology, Baker Heart and Diabetes Institute, Melbourne VIC 3004, Australia;

$22{ }^{11}$ Population Health Science, Bristol Medical School, University of Bristol, Bristol, UK;

$23{ }^{12}$ Medical Research Council Integrative Epidemiology Unit at the University of Bristol, Bristol BS8 1QU,

24 United Kingdom;

$25{ }^{13}$ Computational Medicine, Faculty of Medicine, University of Oulu and Biocenter Oulu, Fl-90014 26 University of Oulu, Finland; 
${ }^{14}$ NMR Metabolomics Laboratory, School of Pharmacy, University of Eastern Finland, FI-70210 Kuopio,

Finland;

$29{ }^{15}$ Department of Epidemiology and Preventive Medicine, School of Public Health and Preventive 30 Medicine, Faculty of Medicine, Nursing and Health Sciences, The Alfred Hospital, Monash University,

31 Melbourne VIC 3004, Australia;

$32{ }^{16}$ CNRS, Pasteur Institute of Lille, University of Lille, 59000 Lille, France;

$33{ }^{17}$ Oulu University Hospital, 90220 Oulu, Finland;

$34{ }^{18}$ Department of Life Sciences, College of Health and Life Sciences, Brunel University London, Uxbridge 35 UB8 3PH, United Kingdom;

$36{ }^{19}$ Centre for Pharmacology and Therapeutics, Department of Medicine, Imperial College London, 37 London W12 ONN, United Kingdom;

$38{ }^{20}$ School of Biosciences and Medicine, Department of Clinical and Experimental Medicine, University of 39 Surrey, Guildford, United Kingdom.

42 \#These authors contributed equally.

$44 \quad *$ Corresponding author: 

diabetes

\section{Abstract}

Type 2 diabetes (T2D) is a global health burden that will benefit from personalised risk prediction and targeted prevention programmes. Omics data have enabled more detailed risk prediction; however, most studies have focussed on directly on the ability of DNA variants predicting T2D onset with less attention given to epigenetic regulation and glycaemic trait variability. By applying machine learning to the longitudinal Northern Finland Birth Cohort 1966 (NFBC 1966) at 31 (T1) and 46 (T2) years old, we predicted fasting glucose (FG) and insulin (FI), glycated haemoglobin (HbA1c) and 2-hour glucose and insulin from oral glucose tolerance test ( $2 \mathrm{hGlu}, 2 \mathrm{hlns}$ ) at T2 in 513 individuals from 1,001 variables at $\mathrm{T} 1$ and $\mathrm{T} 2$, including anthropometric, metabolic, metabolomic and epigenetic variables. We further tested whether the information obtained by the machine learning models in NFBC could be used to predict glycaemic traits in the independent French study with 48 matching predictors (DESIR, N=769, age range 30-65 years at recruitment, interval between data collections: 9 years). In this study, FG and FI were best predicted, with average $R^{2}$ values of 0.38 and 0.53 . Sex, branched-chain and aromatic amino acids, HDL-cholesterol, glycerol, ketone bodies, blood pressure at $\mathrm{T} 2$ and measurements of adiposity at $\mathrm{T} 1$, as well as multiple methylation marks at both time points were amongst the top predictors. In the validation analysis, we reached $R^{2}$ values of $0.41 / 0.55$ for $F G / F I$ when trained and tested in NFBC1966 and 0.17/0.30 when trained in NFBC1966 and tested in DESIR. We identified clinically relevant sets of predictors from a large multi-omics dataset and highlighted the potential of methylation markers and longitudinal changes in prediction.

\section{Key Words}

Glycaemic traits, longitudinal, machine learning, metabolomics, methylation, prediction, type 2 


\section{Background}

Diabetes accounts for the yearly deaths of about four million people between 20 and 79 years old

(2017) world-wide and the prevalence of diabetes is expected to increase from $8.8 \%$ to $9.9 \%$ by $2045 .{ }^{1}$

Moreover, glucose tolerance impairment is progressing in young individuals, leading to high risk of developing type 2 diabetes (T2D) later in life. ${ }^{1}$

To date, T2D risk prediction in the clinical practice has focussed on the classical risk factors of sex, age, obesity, family history, hypertension, cholesterol levels and lifestyle factors ${ }^{2-5}$. Recent advances in omics technologies have allowed exploring the risk factors of T2D in more detail, opening possibilities

for more precise biomarkers and thus, better identification of people at risk for T2D in the future. A large number of metabolites, including amino acids, especially branched-chain amino acids (BRACA) and aromatic amino acids, fatty acids, glycerophospholipids, ketone bodies and mannose have been associated with T2D incidence ${ }^{6-8}$. However, whether metabolites can be effective and reliable T2D predictors, remains unclear.

During the past decade, genome-wide association studies (GWAS) have enlightened the genetic risk of developing T2D. Currently, 403 independent DNA variants are established for T2D risk by GWAS metaanalyses $^{9,10}$ as well as dozens of loci have been associated with quantitative glycaemic traits in individuals without $T 2 \mathrm{D}$, including fasting glucose $(\mathrm{FG})^{11}$, fasting insulin $(\mathrm{FI})^{11}, \mathrm{FG}$ adjusted for bodymass-index $(\mathrm{BMI})^{11}, \mathrm{Fl}$ adjusted for $\mathrm{BMI}^{11}, 2$ hour post-prandial or post oral glucose-tolerance test glucose (2hGluc $)^{12}$ and glycaeted haemoglobin $(\mathrm{HbA} 1 \mathrm{c})^{13}$. Besides genetics, environment and lifestyle are likely to have a large contribution to T2D risk ${ }^{14}$. Environmental factors can affect gene expression by an addition of a methyl group on a CpG-dinucleotide site of DNA. This is called DNA methylation 93 and is the most widely studied type of epigenetic modification ${ }^{15,16}$. Studies in peripheral blood have 94 found a mean absolute difference of $0.5-1.1 \%$ in methylation levels between individuals with and 95 without $\mathrm{T}^{\mathrm{D}} \mathrm{D}^{17}$. Epigenome-wide association studies have reported associations at 65 methylation 
markers for $T 2 D^{17,18}$ and provided support for overlap in epigenetic effects between T2D and glycaemic traits $^{18,19}$. The epigenetic effect on variability of glycaemic traits is magnified in the presence of obesity $^{19}$. The investigation of the link between $\mathrm{BMI}$ and methylation levels demonstrates that methylation at the majority of $\mathrm{CpG}$ sites in blood is consequential to higher $\mathrm{BMI}^{20}$. Interestingly, a weighted methylation risk score calculated from 187 markers was shown to have an even stronger effect on incident $\mathrm{T}^{2} \mathrm{D}^{20}$ than the traditional risk factors of overweight, central obesity, phenylalanine, tyrosine, isoleucine, FG, Fl and C-reactive protein. The methylation risk score remained associated with incident T2D even after adjustment for age, sex, BMI, FI, FG and central obesity ${ }^{20}$.

In the era of multi-omics data and millions of measurable variables, it is challenging to identify the best biomarkers for clinical use. Machine learning approaches are ideal for such high-dimensional data due to their ability to learn from the data and identify patterns without knowledge of the joint distribution 108 of the variables ${ }^{21}$. Recently, the studies of T2D risk have leveraged association analyses as well as 109 machine learning algorithms for the prediction of binary T2D phenotypes. Thus far, machine learning 110 used for T2D classification include logistic regression with and without Lasso regularization ${ }^{17,20,22-26}$, 111 Regularized least-squares $(\mathrm{RLS})^{27}$, Cox regression ${ }^{23}$, naïve Bayes ${ }^{25}$ and J48-decision tree ${ }^{25}$. In predictive 112 studies using machine learning models, classical risk factors, genetic risk scores $(G R S)^{22}$, methylation 113 risk scores (MRS) $)^{17,20}$ and metabolomic data ${ }^{24-27}$ have been used as predictors of T2D incidence after a

114 follow-up window of two to fourteen years. A few studies have suggested that metabolites improve 115 prediction performance ${ }^{23,25-27}$, while others have reported negligible to no improvement in 116 prediction ${ }^{24}$. GRS have been shown to bring no incremental value over classical non-invasive factors 117 and metabolic markers ${ }^{22}$. MRS combining CpG loci have been found to be associated with future T2D 118 incidence ${ }^{17,20}$. All the previous studies have focused on the binary disease status as the outcome which 119 may greatly reduce the power of the analyses. To our knowledge, there are no machine learning 120 studies on predictors of continuous glycaemic traits relevant for T2D pathophysiology. In addition, 121 most of the studies on T2D have used a single pre-selected machine learning approach and have not 
compared their performance in terms of predictive capacities. With the present study, we aimed to address these shortcomings, as well as to shed light on the contribution of longitudinal predictors, especially metabolomic and methylation markers by using data from two well-characterised population-based studies and by applying and comparing six different machine learning approaches.

\section{Results}

We focused on epigenetic and metabolic markers (Supplementary Table 1, Supplementary Table 2)

131 in individuals free from T2D diagnosis or medication at T1. We implemented and compared the results

132 obtained from six machine learning approaches: Boosted trees (BT), Random forest (RF) and support

133 vector regression (SVR) with Linear Kernel with L2 regularization and with L1 and L1/L2 loss functions

134 (SVR-L2Linear-L1, SVR-L2Linear-L1L2, respectively), with Polynomial Kernel (SVR-Polynomial) and with

135 Radial Basis function Kernel (SVR-RBF). The algorithms were chosen for their ability to handle a large

136 number of predictors, to account for multi-collinearity, non-linear relationships, the absence of the assumption regarding data distribution, and for their computational times. We also tested different input data combinations (Figure 1). We further validated our approach in an independent French cohort (Data from an Epidemiological Study on the Insulin Resistance syndrome, DESIR) that shared 48 variables with the NFBC1966 cohort (Supplementary Table 3).

143 We compared the performance of the six models for each of the outcomes (HbA1c, FG, 2hGluc, Fl and

$1442 \mathrm{hlns}$ ) with varying input data combinations, including omics data in their raw or scored forms

145 (Methods, Supplementary Table 4). With the usage of metabolic data as the minimal input, we 
147 metabolic raw data (Mb-R), models with different outcomes were ranked as follows: FI > FG $>2 \mathrm{hlns}>$

$1482 \mathrm{hGlc}>\mathrm{HbA} 1 \mathrm{c}\left(P_{\text {TukeyHSD }}<5.0 \times 10^{-5}\right.$ for all other comparisons except for $2 \mathrm{hlns}>2 \mathrm{~h}$ Gluc for which

$149 P_{\text {TukeyHSD }}=0.19$, Supplementary Table 5). The average coefficients of determination $\mathrm{R}^{2}$ over all machine

150 learning algorithms were $0.53,0.38,0.29,0.25$ and 0.14 (Mb-R) for FI, FG, 2hlns, 2hGlc, HBA1c

151 respectively (Supplementary Table 6).

\section{Best predictor data combinations}

154 Regarding the predictors, we found that models that included at least some metabolic data, either in

155 their raw format (Mb-R) or transformed into scores (Mb-S) (Methods) had the best performance

156 reaching a maximum $R^{2}=0.56$ (Figure $2 A$, Supplementary Table 7 ). In contrast, models with

157 methylation data only as predictors (Mh-R and Mh-S), reached $R^{2}$ values of up to 0.20 . Thus, metabolic

158 models performed significantly better than pure epigenomic models $\left(P_{\text {TukeyHSD }}<1.7 \times 10^{-9}\right)($ Table 1 ,

159 Comparison 1-4; all comparisons are provided in Supplementary Table 8). When metabolic and

160 methylation data were combined, Mb-R performed better for $\mathrm{Fl}$ and $\mathrm{FG}$ than $\mathrm{Mb}-\mathrm{R}+\mathrm{Mh}-\mathrm{R}\left(P_{\text {TukeyHSD }}\right.$

$161<0.04)$. Adding Mh-S to the model did not alter the model performance for any of the outcomes

$162\left(P_{\text {TukeyHSD }}>0.99\right)$ (Table 1, Comparison 5-6). These results suggest that addition of methylation

163 information does not increase the predictive ability of the tested models. When exploring the effect

164 of transforming the original variables into scores (Table 1, comparison 7-8), we observed no significant

165 differences for any of the outcomes when comparing Mb-R vs. Mb-S $\left(P_{\text {TukeyHSD }}>0.29\right)$. This was true also

166 for Mh-R vs. Mh-S, except for the prediction of $\mathrm{FI}$, where Mh-R performed better than Mh-S

$167\left(P_{\text {TukeyHSD }}=1.6 \times 10^{-4}\right)$. Therefore, based on these results we are unable to generalise better performance

168 of the scored data as compared to raw data. Finally, we found that Mb-S + Mh-S model performed

169 significantly better than the model with $\mathrm{Mb}-\mathrm{S}+\mathrm{Mh}-\mathrm{R}\left(P_{\mathrm{TukeyHSD}}<0.02\right)$ for all outcomes except for

1702 hGluc. This observation reflects the decrease in performance of the models upon inclusion of a large

171 number of weak predictors. 


\section{Adjustment for Measures of Adiposity}

174 To understand the influence of the measures of adiposity in the models, we adjusted all the outcomes

175 for T1-BMI, T2-BMI, T1-WHR and T2-WHR and performed the machine learning models on these

176 adjusted outcomes. All adjusted models exhibited an $R^{2}<0.13$ (Figure 2B, Supplementary Figure 1 with

177 a zoomed-in scale for $\mathrm{R}^{2}$, Supplementary Table 9), including models predicting FI and FG. Therefore,

178 the measures of adiposity at $\mathrm{T} 1$ and $\mathrm{T} 2$ are the main drivers of prediction for $\mathrm{FI}$ and FG.

Variable Importance

181 We investigated the contribution of metabolic and epigenomic variables to the prediction of glycaemic

182 traits. We discuss predictors importance only in the context of FG and FI outcomes, for which

183 prediction algorithms reached the best $\mathrm{R}^{2}$ (Figure $\mathbf{2 A}$ ). FG prediction was mostly explained by metabolic

184 variables: valine, leucine, isoleucine, tyrosine, BMI and WHR, HDLs and VLDL, glycerol, alanine, SBP and

185 DBP at T2, WHR and FG at T1 and sex (Figure 3 bottom right). Fl prediction was explained by BMI, WHR,

$186 \mathrm{HDL}$, VLDL, BRACA, phenylalanine, leucine, glycerol, lactate, tyrosine, valine at T2, and FI at T1 (Figure

1873 top left). Once we adjusted the outcomes for the measures of adiposity, the top predictor for FI was

188 measurement of $\mathrm{Fl}$ at $\mathrm{T} 1$, followed by alanine and other variables at $\mathrm{T} 2$ already observed as important

189 before adjustment for measurements of adiposity (Supplementary Figure 2). The top variables driving

190 the prediction of FG were, similarly to FI, valine at T2 and FG at T1 (Supplementary Figure 2). The

191 metabolic models with scored variables were driven by variables that mirrored the top raw predictors.

192 Overall, the model with scored variables for Fl supported the importance of the former variables, as

193 well as ketone bodies (acetoacetate and 3-hydroxybutyrate) at T2 (Figure 3, top right and bottom left).

194 Importantly, even though we previously observed that adding methylation data does not improve

195 model prediction, a number of methylation probes were among the top predictors for both FG and FI

196 when combined with scored metabolic variables (Figure 3). 
Linear regression analyses between $\ln (\mathrm{FI}) / \mathrm{FG}$ and each of the top 25 predictors identified by machine

200 learning indicated that increases in HDL cholesterol were predictive of decreased values of FI and FG

201 (Table 2). The same was true for free cholesterol to total lipids ratio in IDL and cholesterol esters to total lipids ratio in medium VLDL. Similarly, female gender and higher sum score of ketone bodies, i.e. acetoacetate and 3-hydroxybutyrate, predicted lower Fl and FG levels, as did higher methylation levels of the probes T2_cg00574958, T2_cg17058475, and T2_cg08309687. In the models with scored variables, the adjusted $\mathrm{R}^{2}$ for $\ln (\mathrm{FI})$ when including all 25 top predictors was 0.58 . After exclusion of methylation markers, it decreased to 0.54 and finally, after excluding all other variables than measures of adiposity, the variance explained was 0.40 . The same figures for FG were $0.45,0.42$ and 0.31 , respectively.

\section{Prediction from Variables at T1}

211 To test whether variables already 15 years beforehand, i.e. at T1 only, can provide information about 212 glycaemic traits at T2, we restricted the prediction variables to those measured at T1 only. For 213 example, under the well-performing Mb-R in the full model with predictors both from T1 and T2 and 214 unadjusted for measures of adiposity, Fl, FG, 2hlns, 2hGlc, HBA1c were predicted with $\mathrm{R}^{2}$ values of $2150.53,0.38,0.29,0.25$ and 0.14 , respectively. The restriction to $T 1$ variables caused a drop in $R^{2}$ to 0.25 , $2160.22,0.15,0.06,0.06$, respectively, when averaging over all machine learning methods. This suggests 217 that prediction from T1 variables only is not achievable in our dataset.

\section{Performance of the machine learning algorithms}

220 When at least metabolic data in either form (Mb-R or -S + any other data) were included as input, we 221 found no statistically significant differences between the performances of RF and BT for all phenotypes

$222\left(P_{\text {TukeyHSD }}>0.91\right.$, Supplementary Table 10). In addition, no significant difference was found between 
depending on the input dataset. In particular, for datasets with a large number of predictors SVR-

L2Linear-L1L2 was the best performing SVR $\left(P_{\text {TukeyHSD }}<0.05\right.$, Supplementary Table 10). SVR-L2Linear-L1

in turn showed lower performance $\left(P_{\text {TukeyHSD }}<0.05\right.$, Supplementary Table 10$)$ at several occasions when

to minimize MAE. Evaluations based on MAE did not show improved performance of the predictions

\section{Validation of the Machine Learning models in the French DESIR cohort}

240 For the replication analysis, we first trained and tested the data in the NFBC1966 using the set of 48 variables common to both NFBC1966 and DESIR. Next, we trained the data in the NFBC1966 and tested in DESIR. We predicted only FI and FG levels using the top three performing algorithms: RF, BT and SVR-L2Linear-L1L2 (Methods). We were able to predict FI and FG levels in the DESIR data with the average $R^{2}$ values of 0.30 and 0.17 , respectively (Figure 4, Supplementary Table 12). The values were decreased as compared to those when trained and tested in the same data, i.e. NFBC1966 $\left(R^{2}\right.$ for the training data (Methods) produced less variable $R^{2}$ values than those resulting from the use of a 
251 for both FG and FI when tested in the external data, RF and SVR-L2Linear-L1L2 produced smaller

252 decreases than BT, as before (Supplementary Table 12).

\section{Discussion}

256 To our knowledge, this is the first multi-omics study implementing machine learning to predict 257 continuous glycaemic traits over time. We dissected the predictive value of methylation and 258 metabolic, including metabolomic, data from two time points for individual's glycaemic health. We compared six machine learning approaches, while most previous studies have usually focussed on only one selected approach. We detected the best predictive ability of our models for FI and FG levels out of the five glycaemic traits tested, with raw or scored metabolic data as predictors and, BT, RF, or SVRL2Linear-L1L2 as the algorithms. We identified metabolic variables that drove the prediction of the models. We showed that measures of adiposity are the most important contributors to glycaemic health. We also found that methylation probes accounted for 4 and 3 percentage points of the variance explained for FI (58\%) and FG (45\%), respectively. Finally, replication of the approach in an external European descent dataset (DESIR) using a subset of variables common to both cohorts, suggested that

267 RF and SVR-L2Linear-L1L2 are more stable than BT in their performance.

Most of the published studies have targeted T2D onset prediction as a discrete value and rely on the categorization of individuals based on diagnosis thresholds for $\mathrm{HbA1c}, \mathrm{FG}, 2 \mathrm{hGluc}$ and random glucose.

The cut-off points for prediction analyses may vary across studies, and more generally, information

272 loss is rather large when data is categorised ${ }^{28,29}$. Continuous phenotypes, on the contrary, have the

273 potential to reflect the progressive onset of a disease without assuming a discontinuity in the 274 underlying phenomenon. In addition, focussing on the prediction of continuous glycaemic phenotypes 275 themselves allows removing them from the set of predictors for T2D and may reveal more modest

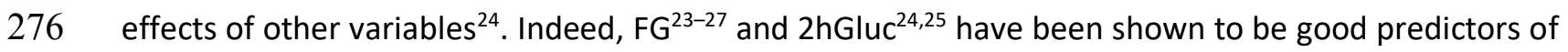


277 T2D. Our study indicated that out of the five glycaemic traits we used, FG and FI were best predictable.

278 This is expected as fasting values are tightly regulated. However, from the clinical practice point of 279 view regarding the prediction of developing T2D, especially FI measurements have less relevance as 280 compared to HbA1c measurements, for example. This suggests that future efforts should be directed 281 towards improving the prediction of the other glycaemic indices than FG and FI.

283 Our study leverages machine learning ability to perform variable selection independently of a pre284 filtering. To date, RLS (a variant of SVR-L2Linear algorithms) ${ }^{27}$, J48-decision tree ${ }^{25}$, and logistic 285 regression with regularization ${ }^{23,26}$ have highlighted the importance of specific metabolites consistent

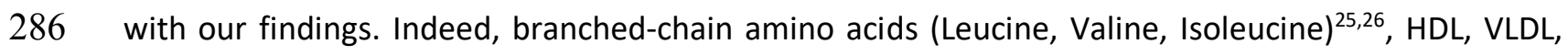
287 glycerol, ApoA and Apo B, 3-hydroxybutyrate ${ }^{26}$, aromatic amino acids (phenylalanine, tyrosine) ${ }^{23,26}$ are 288 established as important predictors by machine learning algorithms, as also shown by our study. 289 Moreover, in this study, we report glycoprotein acetyls and acetoacetate as good predictors of 290 glycaemic trait levels. These markers have previously been associated with T2D ${ }^{30-32}$; however, for the 291 first time here, we show that they are not only associated, but are also predictors of glycaemic health.

In addition to specific metabolites, the machine learning algorithms assigned a high rank (first 25) to several established metabolic health-associated methylation probes in the prediction of FI and FG when collapsing metabolic predictors into scores but keeping methylation probes as such. The probes included for instance those within the genes CPT1A and SREBF1, where the first, CPT1 (Carnitine 297 palmitoyltransferase I) is involved in fatty acid metabolism (RefSeq, Jul 2008), and the latter, SREBF1 298 (Sterol regulatory element-binding transcription factor 1) regulates genes required for glucose 299 metabolism as well as fatty acid and lipid production, and its expression is regulated by 300 insulin ${ }^{33}$. Previously, methylation at CPTIA and SREBF1 has been associated with $2 \mathrm{hlns}^{19}, \mathrm{BMI}^{20}, \mathrm{FG}^{18}$ 301 and $\mathrm{T}^{1} \mathrm{D}^{18}$. In our study, hypomethylation at CPT1A (cg17058475) at T2 predicted higher FI and FG 302 levels, whereas hypermethylation at SREBF1 (cg11024682) at T1 predicted higher FI levels. The 
304 for FG. However, for both glycaemic traits the ranking of 15 methylation probes among the top 25

305 predictors when using scored metabolic data, sets the ground for larger studies of this kind, similarly

306 to the work that has been achieved through large-scale GWAS. A recent study aggregating information over millions of genetic markers into a score showed that genetic risk scores for common diseases can identify people at risk equivalent to monogenic mutations ${ }^{34}$. On that account, the findings from our study encourage further exploration of methylation scores consisting of thousands or even millions of probes in glycaemic trait level prediction.

312 Measures of body adiposity and those of obesity are established risk factors for T2D ${ }^{35}$ and have a well-

313 known impact on glycaemic trait variability ${ }^{36}$. In all six machine learning approaches and within all data

314 combinations, we confirmed the high predictive value of BMI and WHR already 15 years beforehand.

315 Indeed, when we calculated the variance explained from linear regression with the top 25 predictors,

$316 \mathrm{BMI}$ and WHR accounted for the most part of it for both FI ( 0.40 out of 0.58 ) and FG (0.31 out of 0.45$)$.

317 When the outcomes were adjusted for the measures of adiposity, FG and FI levels at T1 gained more 318 weight as predictors. These findings emphasize the importance of classical risk factors in T2D 319 prediction but also show that the tracking is relevant already 15 years beforehand. Taken together, it 320 is clear that classical risk factors will remain as valuable tools in the clinical practice for predicting 321 future glycaemic health. However, more detailed biomarkers, for example certain metabolites as 322 shown in the present study, genetic risk factors as shown recently ${ }^{34}$ and possibly methylation markers 323 will open avenues for more precise prediction.

325 From the algorithm point of view, our analyses showed that the highest prediction performance was 326 achieved with BT, RF and SVR-L2Linear-L1L2 algorithms. This is consistent with the literature as BT and 327 RF have shown to perform well in the prediction studies of various types of data ${ }^{37,38}$. The SVR-L2Linear328 L1L2 (LIBLINEAR library in R) algorithm performed significantly better than the SVR-L2LinearL1 
329 (kernelab library in R). This difference can be explained by the use of the L2-loss over the L1-loss in the

330 SVR-L2Linear-L1L2 in 78.5\% of the cases (i.e. across all external cross-validations, all phenotypes and

331 all data type combinations). We further investigated whether the better performance was due to the

332 choice of the evaluation criterion, namely $R^{2}$ which evaluates the performance in squared terms,

333 similar to L2 loss, rather than in absolute terms, as do MAE and L1. Our analyses showed that SVR-

334 L2Linear-L1 performed worse, regardless of the evaluation criterion used. These observations highlight

335 the importance of loss function choice when using the SVR linear with L2-regularisation, independent

336 of the model performance evaluation criterion. The SVR-Polynomial and SVR-RBF showed slightly

337 lower predictions of the phenotypes, but there were no statistically significant differences when their

338 performances were compared to those of the top three algorithms.

340 Our analysis has some limitations that warrant discussion. First, the relatively small sample size (513

341 subjects) is a drawback for taking full advantage of machine learning prediction with high-dimensional

342 multi-omics data layers. However, our data is the largest to our knowledge featuring both longitudinal

343 data and a comprehensive set of multi-omics data. UK Biobank has expressed its plans to acquire

344 methylation data on its participants ${ }^{39}$. These data will be an important resource for future methylation

345 studies. Nevertheless, the data will be cross-sectional and will not allow investigation of changes in

346 methylation as the data used in the current study data does. Second, parameter tuning and drawing

347 of a threshold regarding variable importance in our machine learning models are not trivial. Longer

348 parameter tuning times might have resulted in more precise predictions and better performance of

349 some or all of the algorithms. Variable importance in turn will depend on the number of variables

350 resampled by the algorithms or the regularization parameters chosen. Overall, we restricted our

351 analyses to six machine learning algorithms in total. It was out of the scope of this study to explore

352 other potentially relevant algorithms, which will remain of future research interest. Third, regarding

353 the samples and the study design, the use of whole blood only for methylation markers, and the

354 relatively young age of the participants, 46 years old at the measurement time of the outcome 
variables is a limitation. The latter might alternatively represent a positive feature, since blood is the

356 easiest tissue to obtain for any study, while the trend of deteriorating glycaemic health in younger

357 adults is growing in all human populations. Finally, our replication effort also has some limitations. We

358 were able to integrate only a small number of variables shared by both cohorts, and the time between

359 the measurements differed between the Finnish and French studies, as did the age of the participants

360 at recruitment. Despite these limitations the tested machine learning models showed promising

361 consistency between the two cohorts, and we would expect better performance would the

362 abovementioned limitations be addressed better.

\section{Conclusions}

365 With the use of six different machine learning algorithms, we have identified clinically relevant sets of

366 predictors of glycaemic traits from large multi-omics datasets and highlighted the potential of 367 methylation markers and longitudinal changes in prediction. In the future, we expect that 368 improvements in study sizes, methylation score computation, finer model tuning and replication in 369 more similar external datasets will improve predictive ability of our models for glycaemic traits and 370 will unveil novel prognostic omics biomarkers for T2D endophenotypes.

\section{Methods}

373 Study Populations

\section{$374 \quad$ NFBC1966}

375 Northern Finland Birth Cohort 1966 (NFBC1966) comprises participants from the two northernmost 376 provinces of Finland with expected dates of birth falling in $1966(\mathrm{~N}=12,058 \text { births })^{40}$. From the medical 377 examination at 31 ( $T 1, N=6,007)$ and 46 years $(T 2, N=5,861)$ we included participants with 378 demographic, medication, epidemiological, blood biochemical, metabolomic and epigenetic 
information available at both time points $(\mathrm{N}=626)$. Consent was obtained and the study was approved by the ethical committees of the University of Oulu and Imperial College London (Approval:18IC4421).

The longitudinal DESIR study (Data from an Epidemiological Study on the Insulin Resistance

The study was approved by the Ethics Committee for the Protection of Subjects for Biomedical

Research of Bicêtre Hospital, France.

\section{Epidemiological, Blood Biochemical and Metabolomic Data in the NFBC1966}

392 Height, weight, waist and hip circumference, and systolic and diastolic blood pressure (SBP, DBP, each 393 measured in triplicate) were measured according to standard study protocols at the clinical 394 examinations at T1 and T2. We used the measured height and weight; however, if unavailable, data 395 from postal questionnaire were used. Body mass index (BMI) was calculated from height and weight and waist-hip-ratio (WHR) from waist and hip measurements accordingly. The biochemical assays ${ }^{43,44}$, oral glucose ${ }^{45}$, and $\mathrm{HbA} 1 \mathrm{c}$ measurements ${ }^{46}$ are detailed elsewhere. Metabolites were quantified by a 398 high-throughput serum nuclear magnetic resonance (NMR) platform ${ }^{47-50}$. Imputation of 399 epidemiological, biochemical and metabolomic variables was performed jointly with random forest 400 (MisForest in $\mathrm{R}^{51}$ ) (Supplementary Methods). Post imputation, for individuals with diagnosed T2D at 401 T2 $(\mathrm{N}=18)$, we corrected their potentially T2D medication-induced and thus artificially normal FG 402 values to $7 \mathrm{mmol} / \mathrm{l}, \mathrm{HbA} 1 \mathrm{c}$ values to $48 \mathrm{mmol} / \mathrm{l}(6.5 \%)$ and $2 \mathrm{hGluc}$ values to $11.1 \mathrm{mmol} / \mathrm{l}$. Detailed 403 descriptions of all the exclusions and corrections are given in the Supplementary Methods. Finally, all 404 the predictor variables were normalised using inverse-normal transformation. The epidemiological, 
Table 1.

\section{Epigenomic Data in NFBC1966}

409 DNA methylation was measured in whole blood from 807 randomly selected individuals after overnight fasting. At T2, DNA methylation was measured for 758 selected subjects that attended the clinical examination, completed the questionnaire and had DNA methylation data from the previous

412 clinical examination available. IlluminalnfiniumHumanMethylation450 Beadchip and EPIC arrays were used at T1 and T2, respectively. Methylation data was quality controlled according to study protocol

(Supplementary Material) and pre-processed on genome build CGCh37/hg19. Imputation of methylation data was performed with random forest (MisForest in $\mathrm{R}^{51}$ ) using the methylation residuals corrected for sex, and blood cell type (Supplementary Material). We limited our analysis to the

417 methylation probes previously associated with seven phenotypes: 187 probes associated with $\mathrm{BMI}^{20}$, 41821 with $\mathrm{FG}^{18}, 11$ with $\mathrm{HbAlc}^{18}$ and 68 with $\mathrm{T}^{12} \mathrm{D}^{18}$, one with $2 \mathrm{hGluc}^{19}$, eight with $\mathrm{Fl}^{19}, 21$ with $2 \mathrm{hlns}{ }^{19}$ (Supplementary Table 2).

\section{Epidemiological, Blood Biochemical and Metabolomic Data in DESIR}

422 A total of 48 predictor variables overlapped between NFBC1966 and DESIR data. These included sex, measures of adiposity, biochemical data (triglycerides, total cholesterol, high and low-density lipoprotein cholesterol (HDL-C and LDL-C) at T1 and T2, insulin and glucose at T1), and metabolomic data (32 variables, 17 at $\mathrm{T} 1$ and 15 at T2). A full list of the included variables is given in Supplementary

Table 3. The data were imputed with the package MisForest in $\mathrm{R}^{51}$ (missingness rate $<1 \%$ )

427 (Supplementary Material). The values of FG were set to $7 \mathrm{mmol} / \mathrm{l}$ if the individual had diagnosed T2D.

428 Finally, all the predictor variables were normalised using inverse-normal transformation. 
432 In total, 513 individuals in the NFBC1966 were included in the machine learning analysis. HbA1c,

433 2hGluc, 2hIns, FG, Fl levels were used as continuous outcomes to predict. A total of 1,001 variables

434 from T1 and T2 were used as predictors in the NFBC1966. Metabolic predictors included:

435 epidemiological data - sex, measures of adiposity (BMI and waist-to-hip ratio), SBP and DBP,

436 biochemical data - ten blood measurements of triglycerides, total cholesterol, high and low-density

437 lipoprotein cholesterol (HDL-C and LDL-C), metabolomic data - 454 metabolites (228 at T1 and 226 at

438 T2) (Supplementary Table 1). The methylation dataset included 528 unique probes, including 264 at

439 T1 and 264 at T2 (Supplementary Table 2).

441 For the replication analysis we chose to predict only FG and FI, for which the predictions worked the

442 best in the NFBC1966 analyses. We included 48 variables common to both cohorts (Supplementary

443 Table 3). The DESIR Cohort did not encompass methylation data. Therefore, before testing the

444 prediction ability of our models in 769 individuals from DESIR, we could train the algorithms in the

445 NFBC1966 either with the 513 individuals as previously, or with all 3,056 individuals who had

446 metabolomics data available. This allowed us to test whether prediction in the external cohort was

447 improved by increasing the sample size of the training data.

\section{Predictor Combinations and Prediction Frameworks}

450 Metabolic $(\mathrm{Mb})$ and Methylation $(\mathrm{Mh})$ data were combined as their individual (or raw, denoted here 451 as R) values or transformed into scores (S) (Supplementary Material). Methylation scores were formed 452 according to the traits the probes have been previously associated with (Supplementary Table 2) and 453 metabolic scores according to specific categories (Supplementary Table 4). We refer to Mb-R/Mh-R 454 when the input data are all represented as individual values and to Mb-S/Mh-S when all the input data 455 are combined in scores. Sex was kept separate and included in the model in addition to the scored 456 variables. The following combinations were tested: Mb-R/ Mb-S/ Mh-R / Mh-S/ Mb-R + Mh-R/Mb-R + 
Mh-S/ Mb-S + Mh-R/ Mb-S + Mh-S (Figure 1). Methylation and Metabolic data were either adjusted

458 for BMI and waist-hip-ratio at T1 and T2, or kept unadjusted.

\section{Machine Learning Approaches}

461 Three machine learning methods were used for regression analysis: Boosted trees (BT), Random Forest

462 (RF) and Support Vector Regression (SVR) (Supplementary Material). SVR was implemented with

463 Linear Kernel with L2 regularization and with L1 and L1/L2 loss functions (SVR-L2Linear-L1, SVR-

464 L2Linear-L1L2, respectively), with Polynomial Kernel (SVR-Polynomial) and with Radial Basis function xgboost in $\mathrm{R}^{51}$ were used with Caret as a wrapper.

\section{Optimization of the Machine Learning Algorithms}

469 Nested cross validation was implemented. The data set was split into a training (80\%) and testing set 470 (20\%) with a 5-fold cross validation. The performance of the machine learning models was estimated 471 on the testing set, while parameter tuning was implemented on the training set by splitting it further 472 into a 5-fold cross validation (nested). Random search method was used to find the model parameter combination (Supplementary Table 13) which minimized the error of the model. The Root Mean Square Error (RMSE) was used to assess model performance during training. Both Rsquared $\left(\mathrm{R}^{2}\right)$ and RMSE were computed in the testing set to estimate performance. For additional checks we used the Mean Absolute Error (MAE) to assess model performance.

\section{Variable Importance in the Machine Learning Models}

479 In BT, the information gain was used as a measure of importance. Gain is based on the decrease in 480 entropy after a dataset is split on a feature $\mathrm{j}$ at a branch of the tree. RF variables were ranked with the 481 Increase in Mean Square Error (MSE). It estimates the increase of prediction error when the values of 
the feature $\mathrm{j}$ are randomly permuted. For SVR, each feature is evaluated based on its independent association with the outcome. The slope of the regression is used to rank the features.

\section{Statistical Analysis for Comparing Model Performance}

486 The performance of each model was computed as the average $R^{2}$ over the 5 testing folds of the cross 487 validation. In the Results section, we report the $\mathrm{R}^{2}$ pooled for the six machine learning algorithms. Comparison of the models was performed with a one-way ANOVA and post-hoc Tukey honest significance test (HSD) test. We use $P$-value $<0.05$ to denote statistical significance.

\section{Association Analysis to Obtain Effect Size, Direction and Variance Explained}

We performed linear regression analyses between the best predicted outcomes ( $\mathrm{FG}$ and $\mathrm{FI})$ and each of the top 25 predictors suggested by the machine learning models to assess the effect sizes and directions of the associations. The analyses were conducted in $\mathrm{R}^{51}$. We report betas with their standard errors and related $P$-values. Additionally, we performed linear regression analyses for $\ln (\mathrm{FI}) / \mathrm{FG}$ by including all the top 25 predictors in the same model and then by removing metabolites/ methylation markers from the set of predictors to evaluate the variance explained and the contribution of metabolites/methylation markers in it. We report the adjusted $R^{2}$ from these analyses.

\section{Replication analysis}

501 For the replication analysis we used the three most consistently performing machine learning approaches: BT, RF, and SVR-L2Linear-L1L2. First, we estimated the performance of the algorithms in the NFBC1966 using a restricted set of 48 predictors. We selected the 48 input variables as overlapping

504 with our validation cohort variables. This performance estimation was done by splitting the NFBC1966 505 dataset of 513 individuals into $80 \%$ for training and $20 \%$ for testing (nested cross validation for tuning 506 as described above). Second, we "re-trained" the model with the maximum number of individuals in 507 the NFBC1966, i.e. 513 individuals which represent $100 \%$ of the previous dataset with methylation and 
metabolomics data, or all 3,056 individuals with metabolomics data only. Then we tested for the ability

509 of this model to predict accurately glycaemic traits when given an independent population (DESIR)

510 with the same input variables. Practically, we used either 513 or 3,056 individuals from the NFBC1966

511 for training and used the resulting models to predict FI and FG in 769 individuals of the DESIR cohort.

512 Performance was evaluated with R2 as described above.

\section{Acknowledgements}

515 IP is funded by the World Cancer Research Fund (WCRF UK) and World Cancer Research Fund 516 International (2017/1641), the Wellcome Trust (WT205915), and the European Union's Horizon 2020

517 research and innovation programme (DynaHEALTH, project number 633595). MAK works in a Unit that

518 is supported by the University of Bristol and UK Medical Research Council (MC_UU_12013/1). The 519 computational work was performed using the Imperial College Research Computing Service, DOI: $520 \quad 10.14469 / \mathrm{hpc} / 2232$

522 We thank all the NFBC cohort members and researchers who participated in the 31 and 46 years study. 523 We also wish to acknowledge the work of the NFBC project center. NFBC1966 31 years old study 524 received financial support from University of Oulu Grant no. 65354, Oulu University Hospital Grant no. 2/97, 8/97, Ministry of Health and Social Affairs Grant no. 23/251/97, 160/97, 190/97, National 526 Institute for Health and Welfare, Helsinki Grant no. 54121, Regional Institute of Occupational Health, 527 Oulu, Finland Grant no. 50621, 54231. NFBC1966 46 years old study received financial support from 528 University of Oulu Grant no. 24000692, Oulu University Hospital Grant no. 24301140, ERDF European 529 Regional Development Fund Grant no. 539/2010 A31592.

531 The D.E.S.I.R. study has been funded by INSERM contracts with Caisse nationale de l'assurance maladie 532 des travailleurs salariés (CNAMTS), Lilly, Novartis Pharma, and sanofi-aventis; INSERM (Réseaux en 533 Santé Publique, Interactions entre les déterminants de la santé, Cohortes Santé TGIR 2008); the 
534 Association Diabète Risque Vasculaire; the Fédération Française de Cardiologie; La Fondation de

535 France; Association de Langue Française pour l'Etude du Diabète et des Maladies Métaboliques

536 (ALFEDIAM)/Société Francophone de Diabétologie (SFD); I'Office national interprofessionnel des vins

537 (ONIVINS); Ardix Medical; Bayer Diagnostics; Becton Dickinson; Cardionics; Merck Santé; Novo

538 Nordisk; Pierre Fabre; Roche; Topcon.

540 The D.E.S.I.R. Study Group. INSERM U1018: B. Balkau, P. Ducimetière, E. Eschwège; INSERM U367: F.

541 Alhenc-Gelas; CHU D’Angers: Y Gallois, A. Girault; Centre de Recherche des Cordeliers, INSERM U1138,

542 Bichat Hospital: F. Fumeron, M. Marre, R Roussel; CHU de Rennes: F. Bonnet; CNRS UMR8090, Lille: A.

543 Fonnebond, S. Cauchi, P. Froguel; Centres d'Examens de Santé: Alençon, Angers, Blois, Caen,

544 Chateauroux, Chartres, Cholet, Le Mans, Orléans, Tours; Institute de Recherche Médecine Générale: J.

545 Cogneau; General practitioners of the region; Institute inter-Regional pour la Santé: C. Born, E. Caces,

546 M. Cailleau, O Lantieri, J.G. Moreau, F. Rakotozafy, J. Tichet, S. Vol.

\section{Author contributions}

549 IP conceived the study idea. IP, MK, LP, HD and ZB defined the phenotypes and quality control criteria.

$550 \mathrm{LP}, \mathrm{HD}, \mathrm{MDA}, \mathrm{MW}$ and MK performed the analyses. LY, BB, RR, SS, MAK, PF and MRJ provided the data.

551 LP, MK and IP formed the central writing group. All authors participated in the critical revision of the 552 manuscript and approved the final version.

\section{Competing Interests}

555 The authors declare no competing interests.

\section{References}

559 1. International Diabetes Federation - Home. Available at: https://www.idf.org/. (Accessed: 31st May 2018) 
2. Buijsse, B., Simmons, R. K., Griffin, S. J. \& Schulze, M. B. Risk assessment tools for identifying individuals at risk of developing type 2 diabetes. Epidemiol. Rev. 33, 46-62 (2011).

563 3. Abbasi, A. et al. Prediction models for risk of developing type 2 diabetes: Systematic literature search and independent external validation study. BMJ 345, 1-16 (2012).

4. Noble, D., Mathur, R., Dent, T., Meads, C. \& Greenhalgh, T. Risk models and scores for type 2 diabetes: Systematic review. BMJ 343, 1243 (2011).

5. Herder, C., Kowall, B., Tabak, A. G. \& Rathmann, W. The potential of novel biomarkers to improve risk prediction of type 2 diabetes. Diabetologia 57, 16-29 (2014).

6. Suhre, K. et al. Metabolic footprint of diabetes: a multiplatform metabolomics study in an epidemiological setting. PLoS One 5, e13953 (2010).

7. Fiehn, O. et al. Plasma metabolomic profiles reflective of glucose homeostasis in non-diabetic and type 2 diabetic obese African-American women. PLoS One 5, e15234 (2010).

8. Drogan, D. et al. Untargeted metabolic profiling identifies altered serum metabolites of type 2 diabetes mellitus in a prospective, nested case control study. Clin. Chem. 61, 487-497 (2015).

9. Scott, R. A. et al. An Expanded Genome-Wide Association Study of Type 2 Diabetes in Europeans. Diabetes 66, 2888-2902 (2017).

10. Mahajan, A. et al. Fine-mapping type 2 diabetes loci to single-variant resolution using highdensity imputation and islet-specific epigenome maps. Nat. Genet. 50, 1505-1513 (2018).

11. Manning, A. K. et al. A genome-wide approach accounting for body mass index identifies genetic variants influencing fasting glycemic traits and insulin resistance. Nat. Genet. 44, 659669 (2012).

12. Saxena, R. et al. Genetic variation in GIPR influences the glucose and insulin responses to an oral glucose challenge. Nat. Genet. 42, 142-148 (2010).

584 13. Soranzo, N. et al. Common variants at 10 genomic loci influence hemoglobin A1C levels via glycemic and nonglycemic pathways. Diabetes 59, 3229-3239 (2011).

586 14. Lowry, E. et al. Understanding the complexity of glycaemic health - Systematic bio- 
psychosocial modelling of fasting glucose in middle-age adults; a DynaHEALTH study. Int. J.

Obes. In press, (2018).

15. Klose, R. J. \& Bird, A. P. Genomic DNA methylation: The mark and its mediators. Trends

16. Cortessis, V. K. et al. Environmental epigenetics: Prospects for studying epigenetic mediation of exposure-response relationships. Hum. Genet. 131, 1565-1589 (2012).

17. Chambers, J. C. et al. Epigenome-wide association of DNA methylation markers in peripheral blood from Indian Asians and Europeans with incident type 2 diabetes: a nested case-control study. Lancet. Diabetes Endocrinol. 3, 526-534 (2015).

18. Walaszczyk, E. et al. DNA methylation markers associated with type 2 diabetes, fasting glucose and HbA1c levels: a systematic review and replication in a case-control sample of the Lifelines study. Diabetologia 61, 354-368 (2018).

19. Kriebel, J. et al. Association between DNA Methylation in Whole Blood and Measures of

601 20. Wahl, S. et al. Epigenome-wide association study of body mass index, and the adverse outcomes of adiposity. Nature $\mathbf{5 4 1}, 81-86$ (2017).

21. Hastie, T., Tibshirani, R. \& Friedman, J. The Elements of Statistical Learning: Data Mining, Inference, and Prediction, Second Edition (Springer Series in Statistics). (2009).

22. Mühlenbruch, K., Jeppesen, C., Joost, H.-G., Boeing, H. \& Schulze, M. B. The Value of Genetic Information for Diabetes Risk Prediction - Differences According to Sex, Age, Family History and Obesity. PLoS One 8, e64307 (2013).

608 23. Yengo, L. et al. Impact of statistical models on the prediction of type 2 diabetes using nontargeted metabolomics profiling. Mol. Metab. 5, 918-925 (2016).

610 24. Savolainen, O. et al. Biomarkers for predicting type 2 diabetes development-Can 611 metabolomics improve on existing biomarkers? PLoS One 12, e0177738 (2017). 
Mellitus to Type 2 Diabetes. Diabetes 65, 2529-2539 (2016).

614 26. Liu, J. et al. Metabolomics based markers predict type 2 diabetes in a 14-year follow-up study. Metabolomics 13, 104 (2017).

27. Peddinti, G. et al. Early metabolic markers identify potential targets for the prevention of type 2 diabetes. Diabetologia 60, 1740-1750 (2017).

28. van Walraven, C. \& Hart, R. G. Leave 'em Alone - Why Continuous Variables Should Be Analyzed as Such. Neuroepidemiology 30, 138-139 (2008).

29. Royston, P., Altman, D. G. \& Sauerbrei, W. Dichotomizing continuous predictors in multiple regression: A bad idea. Stat. Med. 25, 127-141 (2006).

30. Bentley-Lewis, R. et al. Metabolomic profiling in the prediction of gestational diabetes mellitus. Diabetologia 58, 1329-1332 (2015).

31. Akbay, E. et al. The relationship between levels of alpha1-acid glycoprotein and metabolic parameters of diabetes mellitus. Diabetes. Nutr. Metab. 17, 331-335 (2004).

32. Mahendran, Y. et al. Association of ketone body levels with hyperglycemia and type 2 diabetes in 9,398 Finnish men. Diabetes 62, 3618-3626 (2013).

33. Ferre, P. \& Foufelle, F. Hepatic steatosis: a role for de novo lipogenesis and the transcription factor SREBP-1c. Diabetes, Obes. Metab. 12, 83-92 (2010).

34. Khera, A. V et al. Genome-wide polygenic scores for common diseases identify individuals with risk equivalent to monogenic mutations. Nat. Genet. 50, 1219-1224 (2018).

35. Eckel, R. H. et al. Obesity and Type 2 Diabetes: What Can Be Unified and What Needs to Be Individualized? J. Clin. Endocrinol. Metab. 96, 1654-1663 (2011).

634 36. Scott, R. A. et al. Large-scale association analyses identify new loci influencing glycemic traits and provide insight into the underlying biological pathways. Nat. Genet. 44, 991-1005 (2012).

636 37. Caruana, R. \& Niculescu-Mizil, A. An empirical comparison of supervised learning algorithms. 
640 39. Grundberg, E. The opportunities of epigenomic research using UK Biobank data. Available at: http://www.ukbiobank.ac.uk/wp-content/uploads/2018/07/1405-Grundberg.pdf. (Accessed: 10th December 2018)

40. Northern Finland Cohorts. Available at: http://www.oulu.fi/nfbc/. (Accessed: 11th June 2018)

41. Vaxillaire, M. et al. Impact of common type 2 diabetes risk polymorphisms in the DESIR prospective study. Diabetes $\mathbf{5 7}, \mathbf{2 4 4 - 2 5 4}$ (2008).

42. Balkau, B., Eschwege, E., Tichet, J. \& Marre, M. Proposed criteria for the diagnosis of diabetes: (1997).

43. Taponen, S. et al. Hormonal Profile of Women with Self-Reported Symptoms of

44. Taponen, S. et al. Metabolic Cardiovascular Disease Risk Factors in Women with Self-Reported Symptoms of Oligomenorrhea and/or Hirsutism: Northern Finland Birth Cohort 1966 Study. J. Clin. Endocrinol. Metab. 89, 2114-2118 (2004). metabolism in middle-aged men: A follow-up of the Northern Finland Birth Cohort 1966. Prim.

46. Perkiömäki, N. et al. Association between Birth Characteristics and Cardiovascular Autonomic Function at Mid-Life. PLoS One 11, (2016).

47. Soininen, P. et al. High-throughput serum NMR metabonomics for cost-effective holistic studies on systemic metabolism. Analyst 134, 1781-1785 (2009). magnetic resonance metabolomics in cardiovascular epidemiology and genetics. Circ. 
665 49. Wurtz, P. et al. Quantitative Serum NMR Metabolomics in Large-Scale Epidemiology: A Primer on -Omic Technology. Am. J. Epidemiol. (2017).

667 50. Wang, Q., Holmes, M. V., Smith, G. D. \& Ala-Korpela, M. Genetic support for a causal role of insulin resistance on circulating branched-chain amino acids and inflammation. Diabetes Care 40, 1779-1786 (2017).

670 51. R Core Team. R: A language and environment for statistical computing. (2014).

671 
Figure 1. Experimental set-up for Machine learning analysis. We applied machine learning to multiomics data based on blood samples and data collections from the Northern Finland Birth Cohort 1966 at 31 and 46 years. Fasting glucose/insulin (FG/FI), glycated haemoglobin ( $\mathrm{HbA} 1 \mathrm{c})$ and 2-hour glucose/insulin (2hGlu/2hlns) phenotypes at T2 were predicted in 513 individuals using up to 991 variables from T1 and T2: Body-mass-index (BMI), waist-hip-ratio, systolic and diastolic blood pressure (SBP and DBP), sex; 10 blood plasma measurements; 453 NMR-based metabolites; 528 methylation probes established for BMI, FG, Fl, HbA1c, 2hGlu, 2hlns or Type 2 diabetes. Six machine learning approaches were used: random forest, boosted trees and support vector regression (SVR) with the regularization and L1/L2 loss function (L2Linear-L1L2), polynomial and radial basis function (RBF).

Figure 2. Performance as measured in $\mathbf{R}^{2}$ of the different machine learning models. A. Unadjusted for measurements of adiposity (Waist-to-hip-ratio and Body mass index) at T1 and T2; B. Adjusted for measurements of adiposity (Waist-to-hip-ratio and Body mass index) at T1 and T2. Training of the algorithm was performed with a nested cross validation (5-folds outer, and 5-folds inner cross validation) and the $\mathrm{R}^{2}$ of 5 outer testing folds is displayed for each machine learning model. Metabolic predictors include epidemiological, biochemical and metabolomic data. SVR: Support Vector 
699 (var.mod.Imp). Then for each of the six machine learning models, the variables were ranked based on

700 the var.mod.Imp. The rank was averaged over the six models to obtain the "mean variable rank". The

701 latter was used to select top 25 variables for display. For these variables, we display the variable

702 importance after (1) weighting the var.mod.Imp by the $\mathrm{R}^{2}$ obtained for each of the individual machine

703 learning algorithms (2) averaging variable importance across the six machine learning models.

704 FI: Fasting Insulin; FG: Fasting Glucose; RF: random forest; BT: boosted trees, SVR: support vector

705 regression models with the kernels of linear function with L2 regularization and L1 loss function

706 (L2Linear-L1), linear with L2 regularization and L1/L2 loss function (L2Linear-L1L2), polynomial and

707 radial basis function (RBF). Metabolic predictors include epidemiological data, biochemical data and

708 metabolomic data. T2: 46 years old, T1: 31 years old. BMI: Body Mass Index according to clinical

709 examination, postal questionnaire if missing; WHR: Waist-to-hip ratio. Metabolite name descriptions

710 are provided in Supplementary Table 1.

712 Figure 4. Performance as measured in $\mathbf{R}^{2}$ of the different machine learning models with 48 variables

713 shared between NFBC1966 and DESIR. A. Using NFBC1966 data with individuals having methylation

714 data $(\mathrm{N}=513)$ and all available DESIR individuals ( $N=769)$. B. Using NFBC1966 data with all available

715 individuals $(\mathrm{N}=3,056)$ and all available DESIR individuals $(\mathrm{N}=769)$. Training of the algorithm was

716 performed with a nested cross validation (5-folds outer, and 5-folds inner cross validation) and the $\mathrm{R}^{2}$

717 of 5 outer testing folds is displayed for each machine learning model. Metabolic predictors include

718 epidemiological, biochemical and metabolomic data. SVR: Support Vector Regression with the kernel

719 of linear function with L2 regularization and L1/L2 loss function (L2Linear-L1L2). 
722 Table 1. Effect of the input dataset on the prediction performance of the five glycaemic traits.

\begin{tabular}{|c|c|c|c|c|c|c|}
\hline \multirow[b]{2}{*}{ Comparison } & \multirow[b]{2}{*}{ Model } & \multicolumn{4}{|c|}{ Input dataset } & \multirow{2}{*}{$\begin{array}{l}\text { Number of outcome variables for which the } \\
\text { model (A or B) performs the best }\end{array}$} \\
\hline & & Mb-R & Mb-S & Mh-R & Mh-S & \\
\hline 1 & $\begin{array}{l}\mathrm{A} \\
\mathrm{B}\end{array}$ & $x$ & & $\mathrm{x}$ & & $\begin{array}{l}5 / 5, P<2.3 \times 10^{-10} \\
0 / 5\end{array}$ \\
\hline 2 & $\begin{array}{l}\text { A } \\
B\end{array}$ & $x$ & & & $x$ & $\begin{array}{l}5 / 5, P<1.8 \times 10^{-11} \\
0 / 5\end{array}$ \\
\hline 3 & $\begin{array}{l}\text { A } \\
B\end{array}$ & & $x$ & $x$ & & $\begin{array}{l}5 / 5, P<1.7 \times 10^{-9} \\
0 / 5\end{array}$ \\
\hline 4 & $\begin{array}{l}\text { A } \\
B\end{array}$ & & $x$ & & $x$ & $\begin{array}{l}5 / 5, P<6.0 \times 10^{-14} \\
0 / 5\end{array}$ \\
\hline 5 & $\begin{array}{l}\text { A } \\
B\end{array}$ & $\begin{array}{l}\mathrm{x} \\
\mathrm{x}\end{array}$ & & $x$ & & $\begin{array}{l}2 / 5, P<0.04 \text { (equal performance for } 3 / 5 \text { ) } \\
0 / 5\end{array}$ \\
\hline 6 & $\begin{array}{l}\text { A } \\
B\end{array}$ & $\begin{array}{ll}\mathrm{x} \\
\mathrm{x}\end{array}$ & & & $x$ & $\begin{array}{l}0 / 5 \text { (equal performance for all) } \\
0 / 5\end{array}$ \\
\hline 7 & $\begin{array}{l}\text { A } \\
B\end{array}$ & $x$ & $x$ & & & $\begin{array}{l}0 / 5 \text { (equal performance for all) } \\
0 / 5\end{array}$ \\
\hline 8 & $\begin{array}{l}\text { A } \\
B\end{array}$ & & & $x$ & $x$ & $\begin{array}{l}1 / 5, P<1.6 \times 10^{-4} \text { (equal performance for } 4 / 5 \text { ) } \\
0 / 5\end{array}$ \\
\hline 9 & $\begin{array}{l}\text { A } \\
B\end{array}$ & & $\begin{array}{l}x \\
x\end{array}$ & $x$ & $x$ & $\begin{array}{l}4 / 5, P<0.02 \text { (equal performance for } 1 / 5 \text { ) } \\
0 / 5\end{array}$ \\
\hline
\end{tabular}

724 Selected comparisons of models in pairs are displayed to illustrate different scenarios. 1-4) Comparison

725 between models with metabolic or methylation data only; 5-6) The effect of combining two data

726 types.; 7-8) The effect of variable transformations into scores; 9) The decrease in performance of the

727 models upon inclusion of a large number of predictors. Mb-R: Metabolic Raw Variables, Mb-S:

728 Metabolic Scored Variables, Mh-R: Methylation Raw Variables, Mh-S: Methylation Scored Variables.

729 Metabolic predictors include epidemiological data, biochemical data and metabolomic data. FG/FI:

730 Fasting glucose/insulin; HbA1c: glycated haemoglobin; 2hGlu/2hlns: 2-hour glucose/insulin.

731 The performance of all machine learning algorithms upon inclusion of different datatypes was

732 evaluated. For a given phenotype ( $F G, F I, H b A 1 c, 2 h G l u$ or $2 \mathrm{hlns}$ ), the effect of an input dataset was

733 assessed. Column 4 shows which model performed the best, and the number of outcomes for which

734 this pattern is observed. The $P$-value is the maximum $P$-value observed when comparing model $A$

735 against model B for each of the five outcomes. Models were compared with Tukey HSD test following 
bioRxiv preprint doi: https://doi.org/10.1101/358390; this version posted December 13,2018 . The copyright holder for this preprint (which was not certified by peer review) is the author/funder, who has granted bioRxiv a license to display the preprint in perpetuity. It is made available under aCC-BY-NC-ND 4.0 International license.

736 a one-way ANOVA. To test the effect of a given dataset, we run all six machine learning algorithms in

737 a nested cross validation framework ( 5 outer, 5 inner folds), thereby each group compared included

738 six (machine learning algorithms) $x$ five (testing errors) $=30 R^{2}$ measures.

739 
Table 2. Linear regression analysis results for $\ln (\mathrm{FI}) / \mathrm{FG}$ and the top 25 predictors.

\begin{tabular}{|c|c|c|c|c|c|c|c|}
\hline Mb-R + Mh-R & beta & SE & $P$-value & Mb-S + Mh-R & beta & SE & $P$-value \\
\hline $\ln (\mathrm{FI})$ & & & & $\ln (\mathrm{FI})$ & & & \\
\hline T2_BMI & 0.08 & 0.00 & $3.29 \times 10^{-55}$ & T2_MeasuresOfAdiposity & 0.08 & 0.00 & $5.75 \times 10^{-56}$ \\
\hline T2_Large_HDL_FreeChol_\% & -0.11 & 0.01 & $1.48 \times 10^{-40}$ & T2_BRACA & 3.76 & 0.29 & $1.17 \times 10^{-32}$ \\
\hline T2_GlycoproteinAcetyls & 1.32 & 0.10 & $5.88 \times 10^{-37}$ & T1_MeasuresOfAdiposity & 0.07 & 0.01 & $7.39 \times 10^{-26}$ \\
\hline T2_TrigyceridestoPhosphoglycerides & 1.13 & 0.09 & $8.73 \times 10^{-35}$ & T2_BloodProteins & 1.32 & 0.10 & $3.36 \times 10^{-37}$ \\
\hline T2_WHR & 3.79 & 0.27 & $3.12 \times 10^{-38}$ & T2_Carbohydrates & 0.73 & 0.06 & $1.62 \times 10^{-26}$ \\
\hline T2_Phenylalanine & 29.08 & 2.32 & $1.62 \times 10^{-31}$ & T2_cg00574958 & -9.28 & 1.48 & $8.62 \times 10^{-10}$ \\
\hline T2_Large_HDL_FreeChol & -4.91 & 0.39 & $1.03 \times 10^{-31}$ & T2_OtherAminoAcids & 0.66 & 0.18 & $3.42 \times 10^{-4}$ \\
\hline T2_Large_HDL_TotChol_\% & -0.04 & 0.00 & $2.04 \times 10^{-34}$ & T2_cg06898549 & 3.01 & 0.63 & $2.54 \times 10^{-6}$ \\
\hline T2_Isoleucine & 15.32 & 1.23 & $2.52 \times 10^{-31}$ & T2_cg17058475 & -4.67 & 1.12 & $3.45 \times 10^{-5}$ \\
\hline T2_Large_HDL_TotChol & -1.14 & 0.09 & $4.61 \times 10^{-30}$ & T1_CarbohydratesAndInsulin & 0.10 & 0.02 & $1.68 \times 10^{-9}$ \\
\hline T2_Leucine & 13.13 & 1.07 & $1.09 \times 10^{-30}$ & T2_cg08309687 & -3.51 & 0.68 & $3.24 \times 10^{-7}$ \\
\hline T1_FI & 0.80 & 0.07 & $1.19 \times 10^{-27}$ & T2_KetonBodies & -0.70 & 0.29 & $1.52 \times 10^{-2}$ \\
\hline T2_Large_HDL_CholEsters & -1.47 & 0.12 & $1.86 \times 10^{-29}$ & T2_cg10927968 & 3.01 & 0.64 & $3.23 \times 10^{-6}$ \\
\hline T2_Large_VLDL_PhosphoLipids & 4.59 & 0.41 & $1.76 \times 10^{-26}$ & T1_cg08309687 & -2.44 & 0.63 & $1.10 \times 10^{-4}$ \\
\hline T2_Glycerol & 11.03 & 0.94 & $3.94 \times 10^{-28}$ & T2_Lipoparticules & 0.00 & 0.00 & $4.99 \times 10^{-9}$ \\
\hline T2_VLDL_Trigycerides & 0.40 & 0.03 & $6.03 \times 10^{-28}$ & T1_cg26361535 & 1.76 & 0.70 & $1.29 \times 10^{-2}$ \\
\hline T2_Lactate & 0.74 & 0.07 & $2.34 \times 10^{-25}$ & T2_cg25217710 & 4.83 & 1.27 & $1.51 \times 10^{-4}$ \\
\hline T2_Tyrosine & 20.79 & 1.87 & $5.98 \times 10^{-26}$ & T1_cg11832534 & 3.30 & 1.13 & $3.54 \times 10^{-3}$ \\
\hline T2_XL_HDL_PhosphoLipids & -1.30 & 0.11 & $1.81 \times 10^{-28}$ & T1_cg11024682 & 3.92 & 1.02 & $1.33 \times 10^{-4}$ \\
\hline T2_Large_HDL_Trigycerides_\% & 0.10 & 0.01 & $3.53 \times 10^{-26}$ & T1_cg09469355 & -3.98 & 0.93 & $2.11 \times 10^{-5}$ \\
\hline T2_XL_VLDL_Trigycerides & 4.04 & 0.38 & $3.47 \times 10^{-24}$ & T1_cg07728579 & 1.87 & 1.00 & $6.18 \times 10^{-2}$ \\
\hline T2_Valine & 5.83 & 0.54 & $1.48 \times 10^{-24}$ & T2_cg25096107 & -3.36 & 1.40 & $1.68 \times 10^{-2}$ \\
\hline T2_Large_HDL_Particules & -375767.70 & 31515.32 & $4.35 \times 10^{-29}$ & T2_cg26804423 & 4.02 & 1.10 & $2.96 \times 10^{-4}$ \\
\hline T2_XL_HDL_PhosphoLipids_\% & -0.02 & 0.00 & $2.03 \times 10^{-26}$ & T2_cg04524040 & -3.21 & 0.89 & $3.59 \times 10^{-4}$ \\
\hline T2_Medium_VLDL_TotChol & 2.45 & 0.22 & $6.10 \times 10^{-26}$ & T1_BloodProteins & 0.39 & 0.08 & $1.52 \times 10^{-6}$ \\
\hline FG & & & & FG & & & \\
\hline
\end{tabular}




\begin{tabular}{|c|c|c|c|c|c|c|c|}
\hline T2_Valine & 7.43 & 0.56 & $7.64 \times 10^{-35}$ & T2_BRACA & 4.52 & 0.31 & $2.79 \times 10^{-41}$ \\
\hline T2_Leucine & 15.38 & 1.12 & $1.26 \times 10^{-36}$ & T2_MeasuresOfAdiposity & 0.06 & 0.01 & $2.57 \times 10^{-28}$ \\
\hline T2_WHR & 3.73 & 0.30 & $4.59 \times 10^{-31}$ & T1_MeasuresOfAdiposity & 0.06 & 0.01 & $2.56 \times 10^{-14}$ \\
\hline T2_BMI & 0.06 & 0.01 & $8.60 \times 10^{-28}$ & T2_BloodProteins & 1.05 & 0.11 & $1.42 \times 10^{-19}$ \\
\hline T2_Isoleucine & 16.56 & 1.33 & $2.53 \times 10^{-31}$ & Sex & -0.41 & 0.05 & $1.37 \times 10^{-14}$ \\
\hline T2_Tyrosine & 20.74 & 2.05 & $5.73 \times 10^{-22}$ & T2_OtherAminoAcids & 0.96 & 0.19 & $1.08 \times 10^{-6}$ \\
\hline T2_SystolicBloodPressure & 0.01 & 0.00 & $2.82 \times 10^{-20}$ & T1_BRACA & 1.88 & 0.31 & $4.10 \times 10^{-9}$ \\
\hline T1_WHR & 2.80 & 0.30 & $7.01 \times 10^{-19}$ & T1_CarbohydratesAndInsulin & 0.13 & 0.02 & $4.46 \times 10^{-13}$ \\
\hline T1_FG & 0.48 & 0.05 & $1.85 \times 10^{-18}$ & T2_cg11080651 & -8.53 & 1.94 & $1.39 \times 10^{-5}$ \\
\hline T2_Large_HDL_PhosphoLipids_\% & 0.05 & 0.01 & $1.04 \times 10^{-19}$ & T2_cg19695507 & 4.06 & 1.13 & $3.57 \times 10^{-4}$ \\
\hline T2_Large_HDL_FreeChol_\% & -0.09 & 0.01 & $2.80 \times 10^{-20}$ & T2_cg19693031 & -2.79 & 0.73 & $1.53 \times 10^{-4}$ \\
\hline T2_Small_HDL_Trigycerides & 10.63 & 1.52 & $7.58 \times 10^{-12}$ & T2_Lipoparticules & 0.00 & 0.00 & $9.06 \times 10^{-8}$ \\
\hline T2_DiastolicBloodPressure & 0.02 & 0.00 & $1.00 \times 10^{-16}$ & T2_cg26403843 & 2.07 & 0.60 & $6.44 \times 10^{-4}$ \\
\hline T2_Medium_VLDL_Trigycerides_\% & 0.03 & 0.00 & $7.61 \times 10^{-12}$ & T2_cg25217710 & 4.17 & 1.38 & $2.58 \times 10^{-3}$ \\
\hline T2_TrigyceridestoPhosphoglycerides & 0.88 & 0.10 & $1.77 \times 10^{-17}$ & T2_cg09777883 & 2.20 & 1.09 & $4.32 \times 10^{-2}$ \\
\hline T2_Glycerol & 8.74 & 1.08 & $4.71 \times 10^{-15}$ & T1_cg04816311 & 1.75 & 0.67 & $8.93 \times 10^{-3}$ \\
\hline T2_Alanine & 2.89 & 0.35 & $8.41 \times 10^{-16}$ & T2_Carbohydrates & 0.51 & 0.07 & $2.37 \times 10^{-11}$ \\
\hline T2_XL_HDL_Particules & -768070.10 & 98342.58 & $3.27 \times 10^{-14}$ & T1_cg03497652 & 1.96 & 0.70 & $5.47 \times 10^{-3}$ \\
\hline T2_IDL_FreeChol_\% & -0.18 & 0.02 & $2.22 \times 10^{-15}$ & T1_cg11183227 & 3.85 & 1.10 & $5.01 \times 10^{-4}$ \\
\hline T2_Large_HDL_CholEsters & -1.15 & 0.14 & $3.18 \times 10^{-15}$ & T1_cg23906191 & 4.99 & 1.89 & $8.39 \times 10^{-3}$ \\
\hline T2_XL_HDL_CholEsters_\% & 0.02 & 0.00 & $7.27 \times 10^{-12}$ & T1_cg00574958 & -4.21 & 1.64 & $1.06 \times 10^{-2}$ \\
\hline T2_Large_HDL_TotChol & -0.89 & 0.11 & $9.31 \times 10^{-16}$ & T1_cg02059849 & 5.61 & 1.36 & $4.20 \times 10^{-5}$ \\
\hline T2_Medium_VLDL_CholEsters_\% & -0.04 & 0.01 & $8.94 \times 10^{-13}$ & T2_cg00634542 & 2.90 & 1.30 & $2.65 \times 10^{-2}$ \\
\hline Sex & -0.41 & 0.05 & $1.37 \times 10^{-14}$ & T2_cg17058475 & -3.86 & 1.22 & $1.60 \times 10^{-3}$ \\
\hline T2_Small_VLDL_Trigycerides_\% & 0.03 & 0.00 & $1.58 \times 10^{-12}$ & T2_cg06898549 & 2.47 & 0.69 & $3.89 \times 10^{-4}$ \\
\hline
\end{tabular}

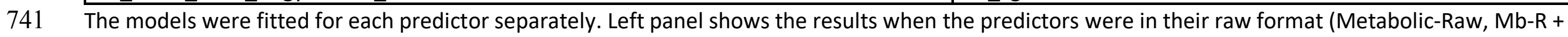

742 Methylation-Raw, Mh-R) and right panel shows when the metabolic predictors were transformed into scores but methylation data kept in raw format

743 (Metabolic-Score, Mb-S + Methylation-Raw, Mh-R). 
bioRxiv preprint doi: https://doi.org/10.1101/358390; this version posted December 13,2018 . The copyright holder for this preprint (which was not certified by peer review) is the author/funder, who has granted bioRxiv a license to display the preprint in perpetuity. It is made available under aCC-BY-NC-ND 4.0 International license.

\section{Figures}

\section{$745 \quad$ Figure 1.}

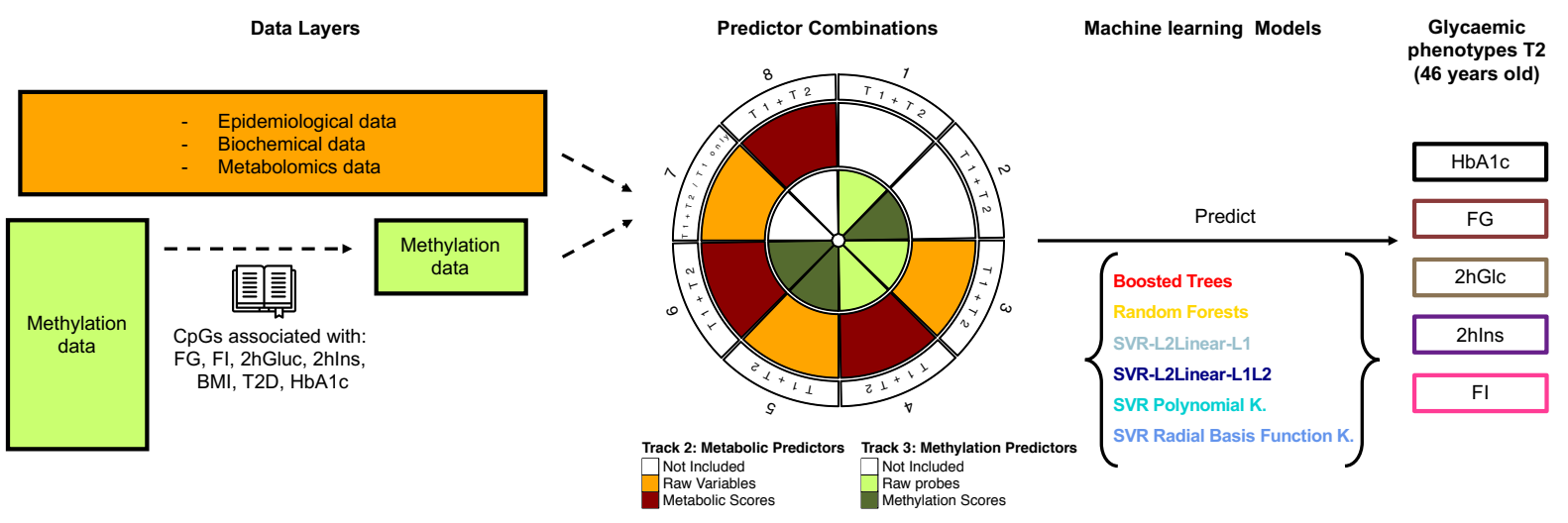

746

Figure 2.

748
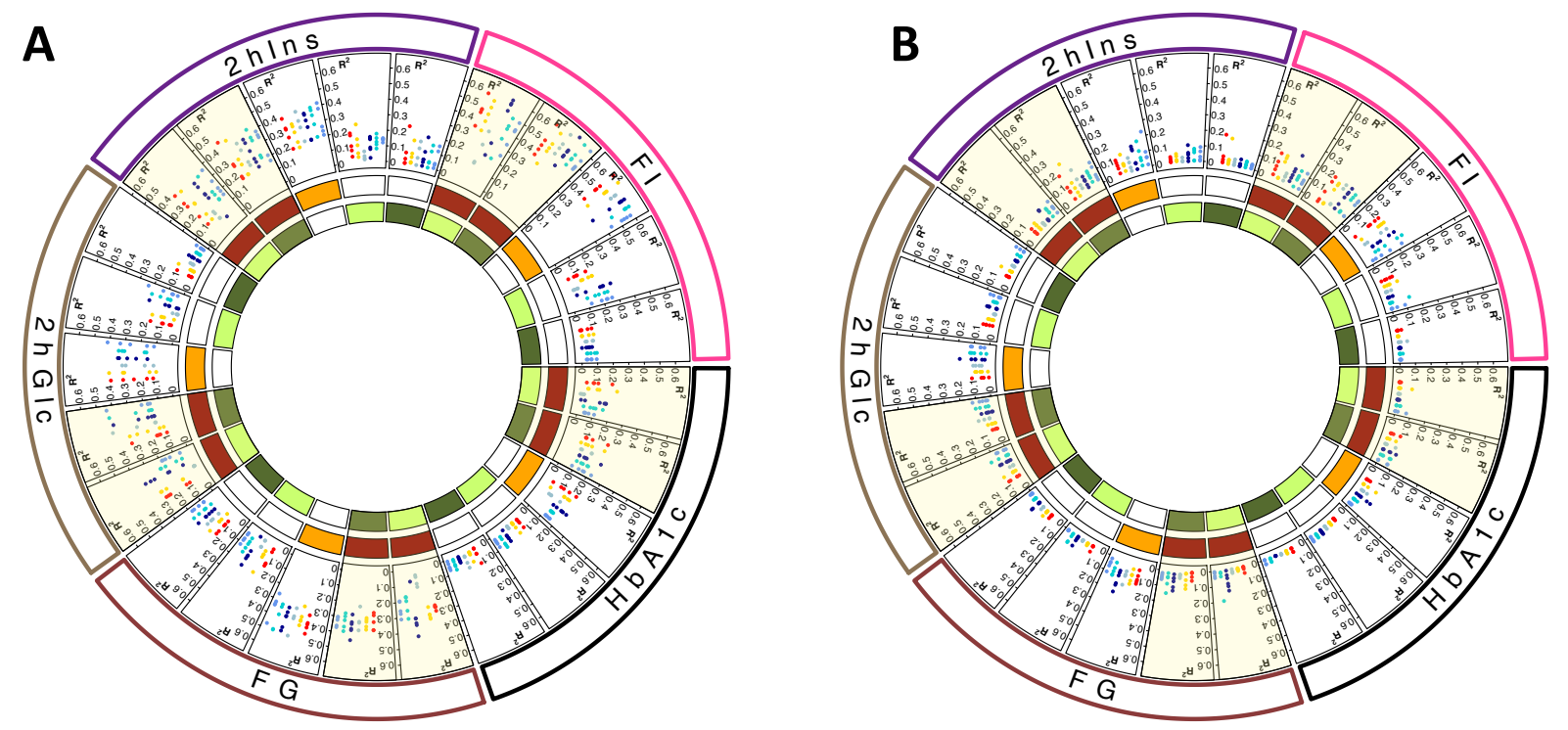

Track 1: Machine Learning Models

- Boosted.Trees

- Random.Forest

- SVR-L2Linear-L1

- SVR-L2Linear-L1L2

- SVR.Polynomial.Kernel

- SVR.Radial.Basis Function.Kernel

Track 2: Metabolic Predictors

$\square$ Not Included

Raw Variables

Metabolic Scores

Overlay: 'Additive' Models

$\square$ Epigenomic \& Metabolic predictors 
bioRxiv preprint doi: https://doi.org/10.1101/358390; this version posted December 13,2018 . The copyright holder for this preprint (which was not certified by peer review) is the author/funder, who has granted bioRxiv a license to display the preprint in perpetuity. It is made available under aCC-BY-NC-ND 4.0 International license.

Figure 3.

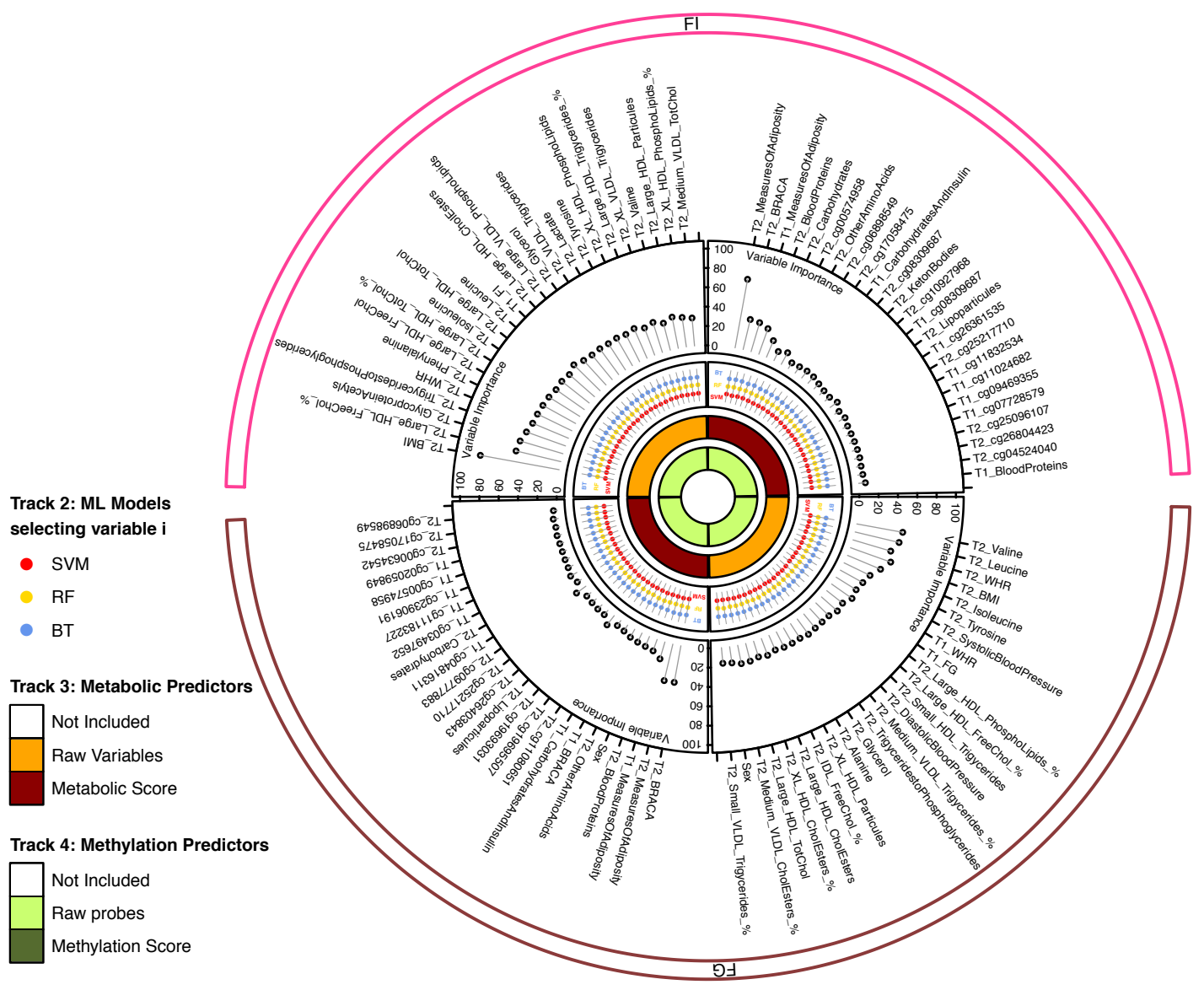

$752 \quad$ Figure 4.
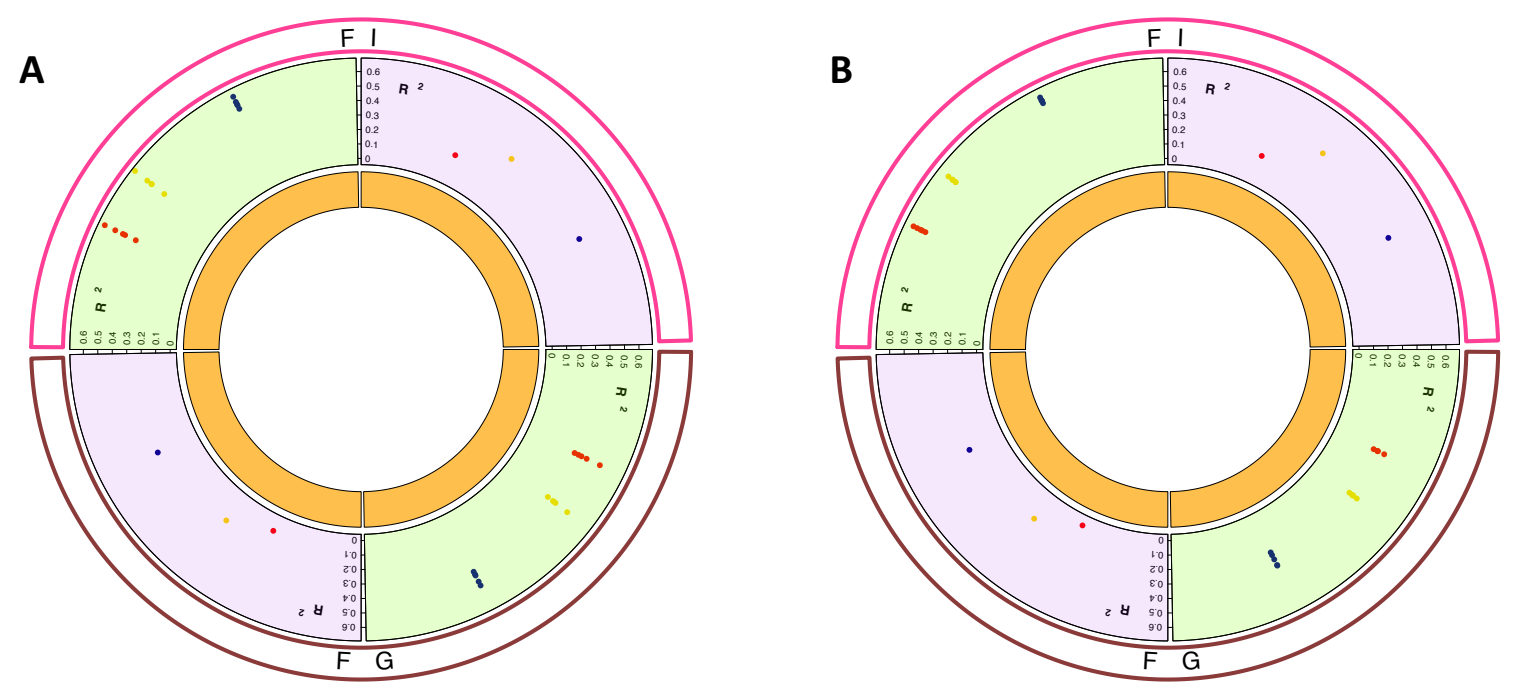

Track 1: Machine Learning Models Track 2: Predictors

- Boosted.Trees

$\square 48$ Metabolic Raw Variables Shared by NFBC and DESIR

- SVR-L2Linear-L1L2

Overlay: Initial performance in NFBC and Replication in DESIR

$\square$ Training NFBC + Prediction NFBC 